\title{
Virtual Simulations in Nursing Training: Using the Coronavirus Pandemic to Shape the Implementation of Innovative Teaching and Learning Methods
}

\author{
Lisa Obermeier ${ }^{1}$, Tanja Jänicke-Stöger ${ }^{2}$ \\ ${ }^{1}$ Technical College of Nursing at Passau Hospital, Passau, Germany \\ ${ }^{2}$ Deggendorf Institute of Technology, Deggendorf, Germany
}

Email address:

l.obermeier@gmx.net (L. Obermeier), tanja.jaenicke-stoeger@th-deg.de (T. Jänicke-Stöger)

\section{To cite this article:}

Lisa Obermeier, Tanja Jänicke-Stöger. Virtual Simulations in Nursing Training: Using the Coronavirus Pandemic to Shape the Implementation of Innovative Teaching and Learning Methods. Clinical Medicine Research. Vol. 10, No. 5, 2021, pp. 159-163. doi: $10.11648 /$ j.cmr.20211005.11

Received: July 28, 2021; Accepted: August 25, 2021; Published: October 29, 2021

\begin{abstract}
Background: The restrictions caused by the pandemic have posed tremendous challenges for those in the healthcare sector, particularly in nurse training. To guarantee continued teaching, course content is being provided via digital learning formats. Objective: Simulation based-learning is an effective learning method used in nursing education. But how can simulation-based learning be carried out virtually? This requires creativity, improvisation and unconventional problem-solving approaches. Methods and results: This experience-based report describes a way in which virtual simulations can be carried out within the context of nurse training at higher education institutions. An example shows how a virtual simulation could be concepted and organized. Initial experiences with the concepted virtual simulations can be reported and positive effects of the learning method as well as problems that have arisen during implementation are presented. Most of the students were able to adapt well to the simulation and achieve a learning effect. Conclusion: Virtual Simulation can be used in a beneficial way in nursing education. Especially simulations with a focus on communication are well-suited to virtual formats so as video-based consulting. Even though video-based consulting in Germany is not yet well-established in all fields, the effects of the pandemic have shown that lots of new approaches can be taken.
\end{abstract}

Keywords: Nursing Education, Virtual Simulation, Digital Learning, Nurse Training

\section{Introduction}

In the Learning and Transfer Centre (LTC) in the "Applied Healthcare Sciences" faculty at the Deggendorf Institute of Technology (DIT), we normally carry out simulation exercises in order to expand on and apply course content with students on the "Nursing (Dual)" programme. Learning on Simulations is an internationally recognized learning method in nursing education with proven learning effects [1, 11]. For this purpose, situations from everyday nursing are taken up and realistically simulated with patient actors. Learning on Simulation aims to enable students to reflect and ultimately to achieve professional skills [14].

The case scenario and the script for the 2020 summer semester had already been prepared and all the dates had been arranged with the tutors, patient actors and students. But the pandemic forced all preparations, considerations and aims related to the simulation training to take a back seat because it was no longer possible to carry out simulations face to face.

Of course, it would have been easy to just cancel these exercises until further notice. The LTC team did not want to settle for this, however. Why should you be unable to carry out a simulation via a virtual medium if you're also planning on delivering lectures in the same way? The idea to carry out virtual simulations was born and put into action straight away.

The documents that had already been produced for the faceto-face simulation were adapted for virtual use and the current situation. This scenario concerns the implementation of "Shared Decision Making" (SDM) within the context of thromboprophylaxis [5]. The primary aim is to apply the concept during a consulting scenario in a domestic setting with a simulation patient using a video conferencing program. 


\section{Adapting the Course Unit to the Virtual Teaching Format}

The first step was providing all necessary information for the students on DIT's learning platform, including a detailed schedule and outline of the virtual simulation process.

They then have the freedom and flexibility to book an appointment from a range of available appointments (morning and evening appointments, in line with shift work) to ensure that the current situation in the healthcare sector is still reflected.

To take part in the virtual simulation, it is essential to brush up on the SDM course content and bring your specialist knowledge of thrombosis (and thromboprophylaxis) up to date. To ensure this, an entrance test (learning platform) was developed and it is necessary to pass this successfully in order to take part in the virtual simulation.

The following table illustrates the process and organisation of a virtual simulation at the LTC:

Table 1. Process and organisation of the virtual simulation.

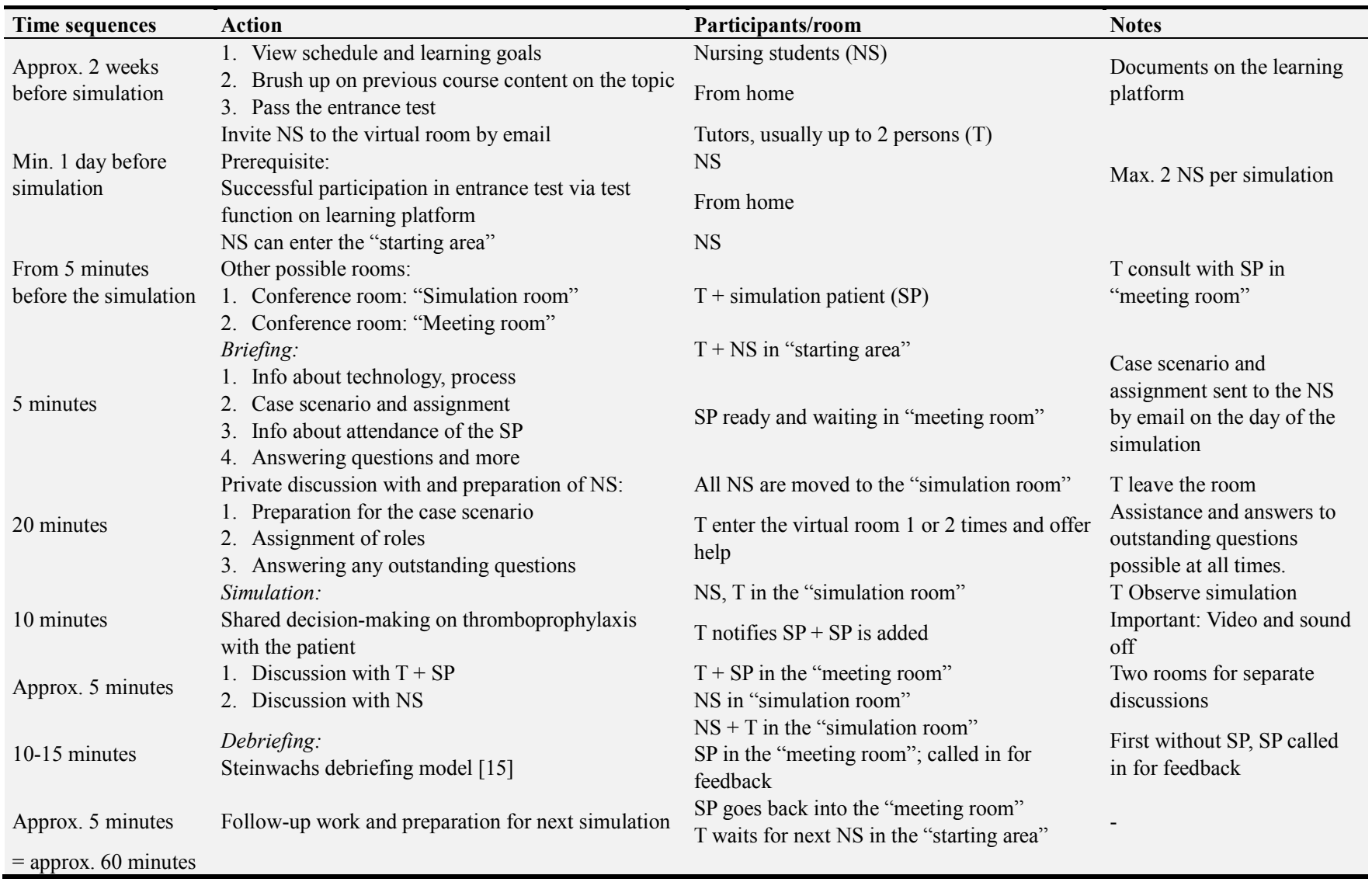

The nursing students first receive the learning goals, the relevant literature and the schedule that they need to carry out the simulation. Tutors then prepare them for the case scenario by giving them a briefing in the virtual room. After a processing and research time of approx. 20 minutes, the consulting scenario with the simulation patient begins. During the simulation in the virtual room, the tutors switch their video and sound off so that they do not distract the students. After the simulation, the tutors have a brief discussion with the simulation patient and the students to share their first impressions and prepare for the subsequent debriefing. Using the Steinwachs model, the debriefing is then held with the nursing students [15] (see Table 1).

The virtual simulation described was carried out using a video conferencing program that enables conference rooms and additional rooms to be set up, so that participants can be assigned to the appropriate rooms.

\section{Experiences of Implementation}

The first step was adapting the case scenario to the current circumstances in health services during the Covid-19 pandemic to make it as realistic as possible. It is therefore stated in the case scenario that the patient was quickly discharged and sent home after she had received initial treatment and her acute symptoms had been stabilised. This is not unusual under the current circumstances and is done in order to achieve a maximum reduction in the risk of infection and to conserve capacity in hospitals. As a result of her short stay in hospital, the patient in the simulation receives outpatient care from nursing staff in the clinical department.

Student involvement in the consulting scenario during the virtual simulation and the handling of the assignment were good. The right atmosphere for discussion was created and 
the students were anxious to complete the task during the simulation. Some students regretted not having prepared themselves better and not having made better use of the time given in terms of consulting.

It was observed that at the beginning of the exercise, the students forgot to greet the simulation patient and ask about her current state of health. It is debatable whether this was due to the unusual virtual scenario or the anticipation (as sometimes observed in face-to-face simulations).

Some students had difficulties responding to specific features of the patient's facial expressions during the virtual scenario. They reported that they weren't able to read these as well as during a face-to-face simulation.

The primary learning effect of this simulation was that the students were required to formulate their specialist knowledge of thromboprophylaxis clearly for the patient. This is a key aspect of practical work and, according to some students, is rather rare in German nursing practice, if it happens at all. This goal can be met in both face-to-face and virtual formats. During the preparation period, the students collected all the relevant information specially for the virtual simulation and, in teams of two, planned how to convey this information. The students used these documents during the simulation. In face-to-face exercises, the students sometimes express inhibitions or uncertainties here with regard to how the use of notes is perceived by the person they are advising.

Tutors expressed fears that this makes them seem less competent. However, experience shows that the documents used in the virtual simulation are not clearly visible to the person being advised and are therefore not considered bothersome. On reflection, it was found that they made students more confident when carrying out the consultation and gave the discussion structure.

It is also noted that observations of the virtual simulation show that the use of notes does not impair the students' expertise or professional manner but in fact only increases these.

Like in a face-to-face simulation, feedback from the simulation patient from the patient's perspective is very valuable for the students. This provides them with feedback on if and what they have understood and how they felt during the simulation. Frequently, the students then ask if and how the patient would be able to make a decision following the consulting scenario and, if necessary, which information or consultancy services were missing in order for them to make a final decision.

The students did not seem wary of the unusual method and means of learning and faced the tutors and the simulation patient in a very open and reflective way. In the event of any technical problems, mostly due to unstable Internet connections, solutions were found together, which enabled the virtual simulations to be carried out as planned (e.g. one student with a good Internet connection calls a second student on their mobile phone so that they can at least take part in the simulation verbally).

The virtual simulation was offered on a voluntary basis. All the more pleasing was that the offer was received positively overall and considered thoroughly advantageous by the students. It provided them with an opportunity to increase their self-confidence and, if necessary, to alleviate any fears about using virtual media. The students reported that during the virtual simulation, they were able to utilise the knowledge they had gained during their university studies, their vocational training at technical colleges and any practical experience they had already gained without even having to be present in person.

Some international examples of implementation are described by Mitchell [12] from London and IIic [8] from Oregon. They report their experiences of using virtual media for nursing and midwifery students to enable them to prepare for practical work in these fields. Mitchell describes the introduction of "online skills sessions" with interactive film materials to prepare the students for a wide variety of patients' health conditions and social circumstances [12]. IIic describes experiences of computer-based, standardised simulations for students in nursing [8]. Both examples of implementation and the review by Coyne et al. also report the observation that students become more self-confident and practised in dealing with virtual simulations and can therefore utilise their skills in a more purposeful way [3].

\section{Looking Ahead}

Expectations of the virtual simulation were not particularly high. Initial thoughts were actually: "A virtual simulation is better than no simulation at all."

In the end, this expectation was more than exceeded. This does not mean that virtual simulations should or will replace face-to-face exercises, but they are a good way of complementing face-to-face exercises, which are time-, labour- and cost-intensive. In virtual simulations, the time and organisational work involved is minimised. It is not strictly necessary to organise as many exercises as possible on one day in order to make optimum use of personnel and spatial resources. For information sessions and consultations and for shared decision-making, the use of virtual simulations actually seems to make sense. The idea of implementing sequences of information sessions and consultations on various topics that are handled during the theory phase (prophylaxis, nursing diagnoses and more), in which students are required to clearly formulate their knowledge for patients and their relatives and then obtain feedback on this, is quite conceivable here.

It seems that simulations with a focus on communication and consulting are particularly well-suited to virtual formats. Nursing and practical technical activities are thus not suited to virtual implementation as it lacks direct contact with patients and any necessary materials (e.g. dressings).

Video-based consulting by caregivers is embraced by patients $[4,7]$ and is already used in various fields, e.g. to improve self-care activities among chronically ill patients [9] or to support families of premature babies [10]. In Germany, telemedical offers were expanded during the pandemic, but there is still no reliable data on the use of these offers [13]. The sound, real-life experience that has already been gained 
could also be used in the future as a basis for other virtual simulations.

The Nursing (Dual) course at DIT has also developed a virtual simulation in which students provide information and advice to patients and their relatives on how to prevent falls among individuals with limited mobility. This simulation is based on the nursing diagnoses "Risk for adult falls" [6] and should students enable to apply scientific findings in everyday practice. It is particularly important to consider the needs of the patient and to express knowledge in an understandable manner. Even though video-based consulting in Germany is not yet well-established in all fields, the effects of the pandemic have shown that lots of new approaches can be taken. Providing information and advice are some of the core duties of nursing and it is therefore hoped that (virtual) consulting will become part of everyday care for our young professionals.

Students at universities can also use the opportunity to practise consulting and advice scenarios virtually via video conferencing in addition to regular face-to-face simulations. For this, there are simulation patients who act as patients or their relative in their own spaces. On the Nursing (Dual) course, the virtual simulations contribute to making a connection between the course content of university studies, technical colleges and practice. On primary qualification nursing courses, the content of university courses can be expanded on and applied in virtual exercises.

Following practical work on the wards, students at technical colleges for nursing receive two weeks of virtual teaching. This decree by the Bavarian State Ministry of Education and Culture applies for all students and also poses a further challenge in addition to the relevant regulations on distance learning, face-to-face teaching and a combination of the two [2]. Simulation-based teaching should not be omitted here in particular. For example, theoretical models of communication that are being taught can be tried out immediately in practice in the form of virtual consulting scenarios with simulation patients, and they can also be expanded on using training videos. These are thus another suitable way of supplementing and expanding on course content, both in these times of distance learning and within the context of face-to-face teaching.

Virtual simulations are used to represent realistic and practical care scenarios without direct contact between patients and their relatives, tutors and students being required. They make a positive contribution to the necessary reduction in contact, which is of crucial importance in the healthcare sector in particular.

\section{Conclusion}

In conclusion, it has to be said that the effects of the Covid-19 pandemic have presented educational institutions with the challenge of implementing new learning formats in everyday teaching in a beneficial way. This should not be seen as an unavoidable burden but used as an opportunity to take innovative, contemporary approaches to the organisation of teaching with the help of digital media.

\section{Declarations}

This article is based on the translation of the paper "Virtuelle Simulationen in der Pflegeausbildung. CoronaPandemie als Wegbereiter zur Umsetzung innovativer Lehrund Lernmethoden", originally published in German in PADUA (2021), 16, 71-74. https://doi.org/10.1024/18616186/a000601. Courtesy of Hogrefe AG, Berne.

\section{References}

[1] Araújo, P, Duarte, T. \& Magro, M. (2018). Effect of Simulation for signifant learning. Journal of Nursing UFPE online, 12 (12), pp. 3416-3425.

[2] Bavarian State Ministry of Education and Culture (Bayerisches Staatsministerium für Unterricht und Kultus) (STMUK) (2020). Retrieved on 13/01/2021 from https://www.km.bayern.de/eltern/schularten/berufsfachschule. html.

[3] Coyne, E., Calleja, P., Forster, E. \& Lin, F. (2021). A review of virtual-simulation for assessing healthcare students' clinical competency. Nurse Education Today, 96, pp. 1-10.

[4] Donaghy, E., Atherton, H., Hammersley, V., McNeilly, H., Bikker, A., Robbins, L., Campbell, J. \& McKinstry, B. (2019). Acceptability, benefits, and challenges of video consulting: a qualitative study in primary care. British Journal of General Practice. 69 (686), pp. 586-594.

[5] Eberhardt, D. \& Obermeier, L. (2021). Decision Coaching Eine simulationsbasierte Lerneinheit im Bachelorstudiengang Pflege (Decision coaching - A simulation-based course unit on the bachelor's programme in nursing). In Kerres, A., Wissing, C. \& Wershofen, B. (ed.), Skillslab in Pflege und Gesundheitsfachberufen (Skills lab in the nursing and healthcare professions). Intra- und interprofessionelle Lehrformate (Intra- and interpersonal teaching formats) (pp. 99-110). Heidelberg: Springer.

[6] Herdman, T., Kamitsuru, S. \& Lopes, C. (2021). Nursing Diagnoses Definitions and Classification 2021-2023. $12^{\mathrm{th}}$ Edition. New York: Thieme.

[7] Husebo, A. M. L. \& Storm, M. (2014). Virtual Visits in Home Health Care for Older Adults. The Scientific World Journal, 2014, pp. 1-11.

[8] Ilic, K. (2020). Schools Transition to Virtual Simulation During Pandemic. Oregon State Board of Nursing, 39 (3), pp. 18.

[9] Levy, S., Henderson, L. \& McAlpine, C. (2014). Growing up with confidence: Using telehealth to support continence selfcare deficits amongst young people with complex needs. Informatics in Primary Care, 21 (3), pp. 113-117.

[10] Lindberg, B., Axelsson, K. \& Öhrling, K. (2009). Experience with videoconferencing between neonatal unit and the families' home from the perspective of certified paediatric nurses. Journal of Telemedicine and Telecare, 15 (6), pp. 275-280.

[11] Mehdipour-Rabori, R., Bagherian, B. \& Nematollahi, M. (2021). Simulation-based mastery improves nursing skills in BSc nursing students: a quasi-experimental study. BMC Nursing (20), pp. 10. 
[12] Mitchell, A. (2020). Pandemic inspires innovative use of virtual simulation to teach practical skills. British Journal of Nursing, 29 (20), pp. 1214.

[13] Scheidt-Nawe, C., Barnes, B., Beyer, A.-K., Busch, M., Hapke, U., Heidemann, C., Imhoff, M., Mumm, R., Paprott, R., Steppuhn, H., von Berenberg-Gossler, P. \& Kraywinkel, K. (2020). Versorgung von chronisch Kranken in Deutschland Herausforderungen in Zeiten der COVID-19-Pandemie. Journal of Health Monitoring. Berlin: Robert-Koch-Institut.

[14] Schröppel, H. (2021) Theoretische Grundlagen zur Methode. In: Kerres, A., Wissing, C., Wershofen, B. (eds) Skillslab in Pflege und Gesundheitsfachberufen. Studium Pflege, Therapie, Gesundheit. Berlin/ Heidelberg: Springer.

[15] Steinwachs, B. (1992). How to facilitate a debriefing. Simulation \& Gaming 23 (2), pp. 186-195.

\section{Biography}

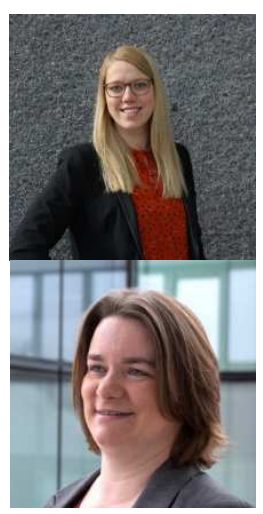

Lisa Obermeier, Nursing Scientist M.Sc., Nurse Educator B.A., Nurse Scientist at Technical College of Nursing at Passau Hospital, Skillstrainer, Freelance Lecturer, Passau, Germany.

Tanja Jänicke-Stöger, Nursing Scientist M.Sc., Nurse Educator B.A., Teacher with Special Duties at Deggendorf Institute of Technology, Deggendorf, Germany. 